\title{
Improved Energy Services Provision through the Intelligent Control of Distributed Energy Resources
}

\author{
M. A. Pedrasa, Student Member, IEEE, E. D. Spooner, and I. F. MacGill
}

\begin{abstract}
There is a need to improve the delivery of energy services, and utilizing distributed energy resources offers significant potential. We propose an energy service modeling technique that would capture temporal variations of its demand and value, and differentiate it from the electric energy consumed by the end-use equipment. We then use this technique with a novel energy service simulation platform that aims to maximize the net benefit derived from energy services. The simulation platform creates a strategy for how available distributed resources should be operated in order to provide the desired energy services while minimizing the cost of consumption. The corresponding optimization problem is solved using particle swarm optimization. The simulation platform proved capable of creating an operation schedule that maximizes net benefit under a range of challenging conditions.
\end{abstract}

Index Terms-Energy services, distributed energy resources, smart home, demand management, particle swarm optimization.

\section{INTRODUCTION}

$\mathbf{E}$ NERGY services are services, processes and commodities from where energy consumers ultimately derive and appreciate the value of raw energy carriers like gas and electricity. Energy services may be classified as either direct or indirect [1]. Direct energy services are services where the raw energy carriers are converted to alternate energy forms that are directly consumed. Examples are illumination, space and water heating, and motion. Indirect energy services are services where the consumer puts value on the processes and products resulting from the utilization of energy carriers and raw materials, if needed. Examples are consumer and manufactured goods, information processing, communications and entertainment.

The value of an energy service originates from the comfort, convenience, products and profits it brings to the consumer. It is affected by several factors like time of the day, weather, social externalities, and uncertainties, among others. In a domestic context, for example, the value of air conditioning is highest during summer but has low value at winter. The value of watching television is certainly very high if an important political or sporting event is in broadcast.

The traditional method of delivering energy services is to generate and make available electricity, regardless of the volume consumed, while ensuring the security of the power

The authors are with the Centre for Energy and Environmental Markets and School of Electrical Engineering and Telecommunications, University of New South Wales, Sydney, Australia (email: m.pedrasa@student.unsw.edu.au).

M. A. Pedrasa acknowledges the scholarship granted by the University of the Philippines (DSF) and the DOST-SEI Engineering Research and Development for Technology Program. system. This approach has become increasingly unfavorable due to growing demand and emissions, and worsening load factors. With these problems facing the power system as a whole, there is a need to improve the efficiency of delivering energy services [1]. The potential of coupling distributed energy resources with existing utility infrastructure to deliver energy services in a more optimal way has been continuously increasing [2].

Distributed energy resources (DER) are fine-grained equipment and practices, usually co-located with or near the consumer, that could augment, or even assume the role of the utility in delivering energy services. In [3], Lovins discussed more than 200 benefits that can be derived from DER and listed a diverse range of its possible forms.

In this paper, we propose an energy service modeling technique that works with a novel energy service simulation platform that would maximize the potential of DER and the utility in delivering energy services. The modeling technique would capture and represent the temporal variations of demand and value of services, and assigns monetary value to the energy that realizes the energy service. The simulation platform aims to improve the provision of energy services by maximizing the net benefits. It would propose a strategy for how available DER should be operated so that services with different values to the consumer are provided while the cost of provision is minimized. The creation of the operation schedule is a challenging optimization problem due of the presence of active and passive storage, and shiftable and curtailable demands.

We will illustrate the potential of the modeling technique and the simulation platform using a smart home as a case study. We will consider DER that are most appropriate in the context of a smart home: renewable energy generation, demand resources or flexible demand, and energy storage. The creation of the strategy is a mathematical optimization problem, and we used a hybrid of particle swarm optimization and its binary version to find the solution.

Several similar works have recently presented techniques on how to optimize the operation of DER. The household energy consumption is minimized in [4] by planning the operation of space heaters and shiftable loads using tabu search and multi-agent systems. In [5], electricity and natural gas, and co-generation technologies have been used to service heating and electricity end-use loads.

The rest of the paper is outlined as follows: The proposed energy service modeling technique and simulation platform are discussed in Sections II and III. Particle swarm optimization is described in Section IV. The case study is presented in Section $\mathrm{V}$. The conclusions are summarized in Section VI. 


\section{Modeling Energy Services}

The demand for and the value of energy services change with time. In a restaurant, for example, hot water is generally in demand an hour or two before it opens, while it is open, and an hour or two after it closes. Hot water is generally not needed beyond those hours. Furthermore, the convenience of having hot water flowing out of the taps is certainly higher during those hours, than when no one is using it. It may be argued that the restaurant owner is willing to pay more money per 1 unit of thermal energy (MJ or Btu) needed to heat the water when the restaurant is open than when it is closed.

In this illustration, the value of the hot water energy service is assigned to the thermal energy content of water, instead of assigning it to the energy consumption of the heater. The workers benefiting from the hot water service are aware of the convenience of using hot water, and would not care about the number of $\mathrm{kWh}$ of electricity consumed by the heaters.

The value of a service is the monetary amount that the user is willing to pay so that the service is provided. It could also be the amount that the user would lose if the service is not provided. The monetary value, therefore, is perceived or may be computed by the user.

In the proposed modeling technique, the value of the service is assigned to each unit of energy that realizes the service. By doing so, we can make a distinction between that energy and the electric energy consumed by the equipment that delivers the service. The energy that realizes the energy service, whether it is mechanical, thermal or luminous in nature, could be referred to as the "energy equivalent" of the energy service.

Putting the value on the "energy equivalent" also enables us to differentiate between different end-use equipment. To illustrate, two different heaters may introduce the same amount of thermal energy to water, but consume different amounts of electric energy.

The demand for and the value of hot water service for the restaurant example, therefore, may be modeled as a pair of time-series values. The first series describes the hourly demand for the hot water service. It may be the hourly consumption of hot water specified in liters, or the required hourly heat content of water (the "energy equivalent" of the hot water service, $\left.U_{E S}(t)\right)$ in $\mathrm{kWh}$. The second series, $\lambda_{E S}(t)$, describes the hourly variation of the monetary value that the owner assigns to the "energy equivalent" of the hot water service per unit of thermal energy.

These temporal variations can be better visualized using graphs instead of time-series. As an illustration, the demand and value of the hot water service may be described by the plots in Fig. 1. The plots show that the demand for hot water during the opening hours is higher than outside of those hours, and the value of hot water is also higher during those hours. There could be hours when consumption is low but value is high, and on some hours, the consumption can be high yet the value is low. The amount of $U_{E S}(t)$ depends on the consumption habits of the user. Its monetary value, $\lambda_{E S}(t)$, is perceived or may be computed by the user.

In some cases, it is not possible to depict the demand for an energy service as an hourly variation of some variable

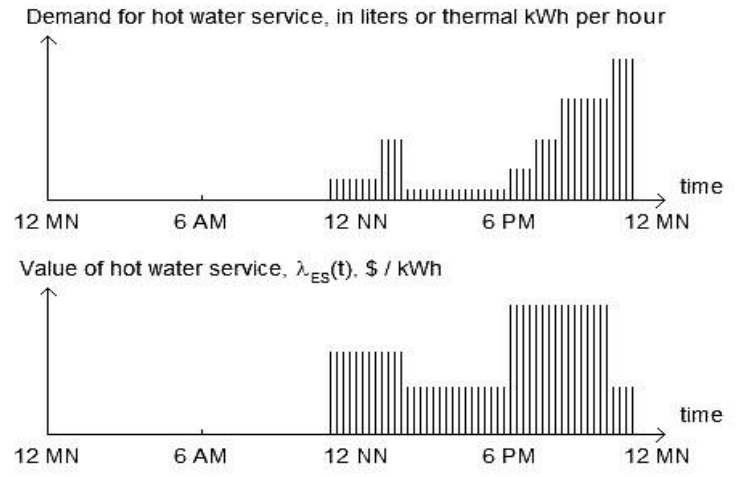

Fig. 1. Representation of energy service demand and value.

or of the "energy equivalent," like what is shown in Fig. 1. Examples are shiftable services, like cooking or washing, and interruptible services, like pool pumping. The timing of delivery of these services are flexible, that is, the start times and durations may be variable, and they may be interrupted and the rest of the service may be postponed to a later time. In such services, a narrative description of the demand may be given, for example, "the washing service requires $1 \mathrm{~kW}$ of electricity over a continuous two hour period, and may start anytime between 9 AM and 3 PM."

The relationship between the "energy equivalent" of a service, $U_{E S}(t)$, and the electric energy consumed by the enduse equipment, $P_{e}(t)$, should be determined for each service to be provided. The relationship heavily depends on the physical processes occurring within the equipment and the service itself.

The determination of the "energy equivalent" is straightforward if the end-use equipment instantaneously converts electricity to the end-use energy or process. Examples of such equipment are light bulbs and appliances. If the efficiency of the equipment in converting electricity to the energy service is $\eta$, then,

$$
U_{E S}(t)=\eta \cdot P_{e}(t) .
$$

In some types of energy services, the conversion from electricity to energy service is not instantaneous, that is, there is some form of storage involved. Therefore, there is a temporal mismatch between the demand for a service and the electricity consumption of the end-use equipment that delivers the service. Examples of such services are space heating and storage water heating. To illustrate, in storage-type water heating, water is heated overnight but it is consumed over the entire day. The relationship between the "energy equivalent" of space heating service and the energy consumption of the heater will be illustrated in the case study in Section V.

There is no perceptible "energy equivalent" or it is difficult to quantify for indirect energy services. In this case, the actual electric energy consumption may be assigned as the "energy equivalent" of the service.

\section{Energy Service Simulation Platform}

The energy service simulation platform we propose aims to maximize the net value of the energy services desired by the 
consumer. The net value is equal to the total benefits derived from the availability of the services less the cost of electricity consumption. The simulation platform takes advantage of the following points:

1) Temporal variation of the cost of electricity, e.g. Time of Use and Real-Time Tariffs.

2) Flexible delivery of some types of energy services.

3) Temporal mismatch between energy service demand and electricity consumption of end-use equipment.

4) Availability of active storage DER like battery banks.

The platform would propose a strategy for how available DER should be operated. The strategy aims to minimize the cost of electricity consumption while delivering the required services. The strategy will be in the form of a schedule, or a set of recommended actions at each interval of the simulation horizon. The platform will also quantify the savings incurred by operating the DER using the strategy, and this result may be used for making investment decisions.

The simulation platform is basically a mathematical optimization problem expressed as

$$
\max \sum_{t=1}^{T}\left(\left(\sum_{i=1}^{S} \lambda_{E S, i}(t) U_{E S, i}(t, \boldsymbol{x})\right)-\lambda_{e}(t) P_{e}(t, \boldsymbol{x})\right)
$$

where

$T$ number of hours in the simulation period,

$S \quad$ number of energy services,

$\lambda_{E S, i} \quad$ value of the "energy equivalent" of the $i^{t h}$ service $(\$ / \mathrm{kWh})$,

$U_{E S, i} \quad$ demand for the "energy equivalent" of the $i^{t h}$ service $(\mathrm{kWh})$,

$\lambda_{e} \quad$ cost of electricity $(\$ / \mathrm{kWh})$,

$P_{e} \quad$ total hourly electricity consumption $(\mathrm{kWh})$, subject to the operational constraints of the DER.

The optimization problem aims to find the schedule of operation of the DER, $\boldsymbol{x}$. The first term in (2) is the total benefit derived from the availability of the services, computed using the monetary values assigned to the "energy equivalent" and demand for the services. The second term is the total cost of electricity used to deliver the services.

The complexity introduced by passive storage to the relationship between the energy consumption and "energy equivalent," and the presence of complex DER operation models and active storage options like battery banks suggest that the objective function in (2) is non-linear, non-convex and noncontinuous. Simulation-based heuristic techniques, therefore, offer great potential in finding the optimal or a near-optimal solution. We used particle swarm optimization as the optimization tool.

\section{PARTICle SWARM Optimization}

Particle swarm optimization (PSO) is a population-based search technique that mimics how a group of simple particles could achieve complex collective behaviors [6]. Each particle in a swarm represents a solution to the optimization problem, and the particles search for the optimal solution by flying around the solution space while communicating with each other. The trajectory of a particle is affected by the best performing particle and by the best position it has visited. The movement of a particle is described by its speed $V\left(v_{1}, v_{2}, \ldots, v_{n}\right)$ and position $P\left(p_{1}, p_{2}, \ldots, p_{n}\right)$. The $i^{t h}$ coordinate of speed and position are computed by

$$
\begin{gathered}
v_{i}^{t+1}=\omega \cdot v_{i}^{t}+c_{1} \cdot \operatorname{rand}() \cdot\left(p_{\text {Gbest }, i}^{t}-p_{i}^{t}\right) \\
+c_{2} \cdot \operatorname{rand}() \cdot\left(p_{\text {Pbest }, i}^{t}-p_{i}^{t}\right) \\
p_{i}^{t+1}=p_{i}^{t}+v_{i}^{t+1} .
\end{gathered}
$$

In (3), the subscripts Gbest and Pbest refer to the position of the best performing particle (global best) and the best position that the particle has visited (personal best). The first term is the momentum, while the last two terms are the weighed pull of the global and personal best positions. $\operatorname{rand}()$ is a uniform random number generator from 0 to 1 .

The particles are placed randomly in the solution space in the initialization phase. The initial particles may be preprocessed to accelerate convergence and increase the chances of finding the optimal solution. The particles then fly around the solution space, as determined by (3) and (4). The global and personal bests are updated if needed, and the selection is determined by evaluating the objective function. The simulation stops when a convergence criterion has been satisfied or the maximum number of iterations has been reached. The global best particle at the end of the simulation is taken as the solution to the problem.

The binary version of PSO (BPSO) was proposed in [7] to solve binary-valued optimization problems. In BPSO, a coordinate of a particle position, $p_{i}$, is either 0 or 1 . The speed is also computed using (3), however, it is restricted to be within a range, $\left[-V_{\max }, V_{\max }\right]$. The position is computed by mapping the velocity to a probability using a sigmoid function (5) and the result is compared to a random number generator. That is, if $\operatorname{rand}()<S\left(v_{i}^{t+1}\right)$, then $p_{i}^{t+1}=1$, otherwise, $p_{i}^{t+1}=0$.

$$
S\left(v_{i}^{t+1}\right)=\frac{1}{1+\exp \left(-v_{i}^{t+1}\right)} .
$$

PSO and BPSO and their variations have been shown to be effective in generating near-optimal solutions to complex optimization problems [8]-[10].

\section{CAse Study}

\section{A. Energy Service Provision in a Smart Home}

The energy service simulation platform was used to determine how DER may be controlled in a smart home. The available DER are:

1) Heater: for space heating service; maximum heating power equal to $2.0 \mathrm{~kW}$; resistive type.

2) Pool pump: for pool maintenance; rated $1.0 \mathrm{~kW}$.

3) Battery storage: $2.4 \mathrm{kWh}$ capacity; $400 \mathrm{~W}$ maximum charging and discharging rates; $90 \%$ charging and discharging efficiency; $0.1 \%$ coulomb loss per hour; may be discharged down to $20 \%$ of capacity.

4) PV array: $2.0 \mathrm{kWp}$.

All energy services aside from space heating and pool pumping are lumped together into a must-run aggregate energy service. The demand for the must-run service is shown in Fig. 


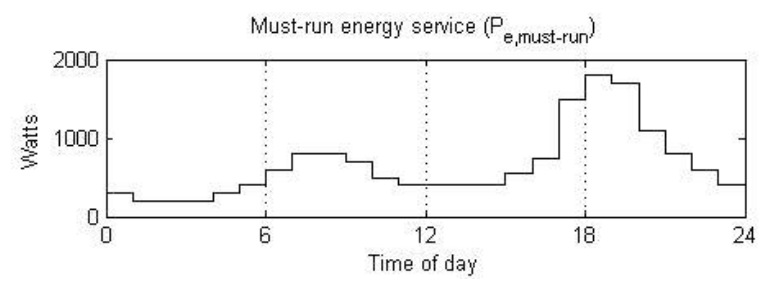

Fig. 2. Demand for must-run service.

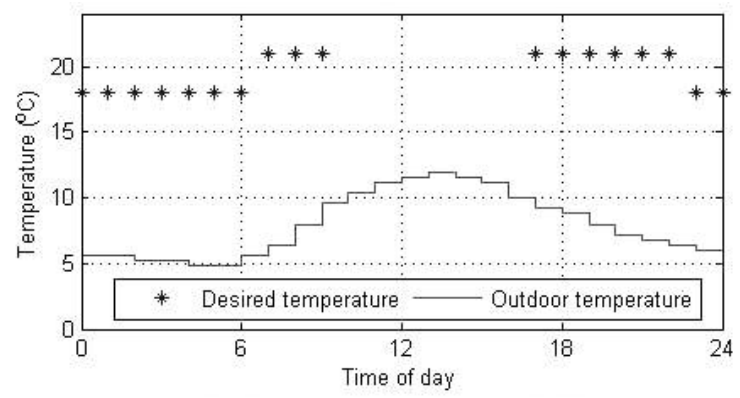

Fig. 3. Demand for space heating service.

2. For the space heating service, the resident is comfortable as long as the room temperature is within $1 \mathrm{C}^{\circ}$ of the desired value. The desired and outdoor temperatures are shown in Fig. 3. The resident leaves the house at $9 \mathrm{AM}$ and returns at $5 \mathrm{PM}$, so there is no indicated desired value in that period. The pool pump should be operated at most 6 hours a day. The pump can be run in two shiftable 3-hour periods anytime between 8 $\mathrm{AM}$ and $10 \mathrm{PM}$.

The simulation platform will generate a strategy for how the first three DER should be controlled. For each hour of a 24-hour period, it will determine (a) the heating power, (b) whether a 3-hour pool pumping period should be started, and (c) the charging or discharging rates of the battery.

Except for the baseline case, a strategy will be derived for each of the scenarios summarized in Table I. In all cases, the house is under Time-of-Use rates. The electricity rates are summarized in Table II. These are the actual rates in Sydney, Australia (ToU) [11] and South Australia (net feed-in) [12] as of July 2008.

In the baseline case, the resident manually controls the DER. He raises the thermostat to $21^{\circ} \mathrm{C}$ at $6 \mathrm{AM}$ so that by $7 \mathrm{AM}$, the desired temperature is achieved. He also reduces the setting from $21{ }^{\circ} \mathrm{C}$ to $18{ }^{\circ} \mathrm{C}$ at $10 \mathrm{PM}$, an hour before the desired $11 \mathrm{PM}$ reduction. To ensure that the temperature is within the desired range when he arrives at $5 \mathrm{PM}$, he programs the heater to turn on at $3 \mathrm{PM}$ with the thermostat set to $21^{\circ} \mathrm{C}$. He programs the pool pump timer so it would run from 9 AM to 3 PM, during the period when the PV output is high. There are no batteries installed, and any energy export is compensated by the retailer at $\mathrm{ToU}$ rates.

To demonstrate the value of scheduling and to determine if the installation of batteries would be a sound investment, we used the simulation platform to create a DER operation strategy for Cases 2 to 6 . In Cases 2 and 4, only the heater and pool pump are controlled, and net feed-in tariff is available in Case 4 . The value added by the battery storage is determined in
TABLE I

DESCRIPTION OF SCENARIOS

\begin{tabular}{c|l}
\hline Case number & Description \\
\hline 1 & $\begin{array}{l}\text { Baseline case. Manual control of DER. No battery } \\
\text { storage. }\end{array}$ \\
\hline 2 & DER are scheduled. No battery storage. \\
\hline 3 & DER are scheduled. With battery storage. \\
\hline 4 & $\begin{array}{l}\text { DER are scheduled. With net feed-in tariff. No battery } \\
\text { storage. }\end{array}$ \\
\hline 5 & $\begin{array}{l}\text { DER are scheduled. With net feed-in tariff and battery } \\
\text { storage. }\end{array}$ \\
\hline 6 & $\begin{array}{l}\text { DER are scheduled. No battery storage. Value of pool } \\
\text { pump service from 8AM to 10PM is reduced from } \\
\text { medium to low. }\end{array}$ \\
\hline
\end{tabular}

TABLE II

ELECTRICITY TARIFF

\begin{tabular}{l|c}
\hline Tariff type & Cost, $\lambda_{e}(\$ / \mathrm{kWh})$ \\
\hline Time of Use & \\
Peak (2-8 PM) & 0.3025 \\
Shoulder (7AM-2PM, 8-10PM) & 0.1089 \\
Off-peak (10PM-7AM) & 0.0605 \\
\hline Feed-in rate (net) & 0.440 \\
\hline
\end{tabular}

Cases 3 and 5. In Case 6, the value of the pool pumping service is reduced from medium to low. This case will demonstrate how a different perception to the value of a service could affect its provision.

\section{B. Energy Service Models}

We must model the temporal variations of the demand for and value of the three services to be provided (must-run, space heating and pool pumping) before we can use (2) to generate the schedule. For the must-run service, the actual energy consumption shown in Fig. 2 is assigned as the "energy equivalent" of that service, that is,

$$
U_{E S, \text { must-run }}(t)=P_{e, \text { must-run }}(t) \text {. }
$$

The demand for the space heating service is described by the desired hourly temperature shown in Fig. 3. The "energy equivalent" of the space heating service is the thermal energy content of the indoor air when it is at the desired temperature. This thermal energy is equal to the heating load of the building. The heating load is equal to the heat losses through the building enclosure and external air infiltration [13]. Infiltration is ignored, so the heating load is equal to

$$
\begin{aligned}
Q(t) & =\frac{1}{R}\left(\theta_{\text {des }}(t)-\theta_{\text {out }}(t)\right) \\
& =U_{E S, \text { heat }}(t)
\end{aligned}
$$

where

$Q \quad$ heating load = "energy equivalent" of space heating service,

$R \quad$ thermal resistance of the building shell $=16 \mathrm{C}^{\circ} / \mathrm{kW}$,

$\theta_{\text {des }}$ desired temperature $\left({ }^{\circ} \mathrm{C}\right)$,

$\theta_{\text {out }}$ outdoor temperature $\left({ }^{\circ} \mathrm{C}\right)$.

We assumed that the heating service is delivered if the actual temperature is within $1 \mathrm{C}^{\circ}$ from the desired temperature. The 
TABLE III

Monetary Value Equivalent of the Perceived Value of SERVICES

\begin{tabular}{c|c}
\hline Perceived value of service & Monetary value $(\$ / \mathrm{kWh})$ \\
\hline High & 0.60 \\
Medium & 0.20 \\
Low & 0.08 \\
No value & 0.00 \\
Expense & -0.50 \\
\hline
\end{tabular}

actual indoor temperature, $\theta_{i n}$, may be solved by using $\theta_{i n}$ in (7) and combining the resulting equation with (8) to get (9). (8) relates the heat introduced to the air to the change in temperature.

$$
\begin{gathered}
C \frac{d \theta_{\text {in }}(t)}{d t}=P_{\text {heat }}(t)-Q(t) \\
C \frac{d \theta_{\text {in }}(t)}{d t}=P_{\text {heat }}(t)-\frac{1}{R}\left(\theta_{\text {in }}(t)-\theta_{\text {out }}(t)\right)
\end{gathered}
$$

In (8) and (9), $C$ is the heat capacity of indoor air $=0.525$ $\mathrm{kWh} / \mathrm{C}^{\circ}$, and $P_{\text {heat }}$ is the heating power. The discrete-time equivalent of (9) using 1-hour time-steps is

$$
\begin{aligned}
\theta_{\text {in }}(t+1) & =\theta_{\text {in }}(t) e^{-\Delta / \tau}+R P_{\text {heat }}(t)\left(1-e^{-\Delta / \tau}\right) \\
& +\theta_{\text {out }}(t)\left(1-e^{-\Delta / \tau}\right)
\end{aligned}
$$

where $\Delta=1$ hour and $\tau=R C$.

We assumed that the heater energy consumption is entirely converted to heat so

$$
P_{e, \text { heat }}(t)=P_{\text {heat }}(t) .
$$

The pool pump should run at most 6 hours a day, anytime from $8 \mathrm{AM}$ to $10 \mathrm{PM}$ and may be run as two shiftable 3hour periods. The actual energy consumption of the pump is assigned as the "energy equivalent" of the pool pumping service, or

$$
U_{E S, p o o l}(t)=P_{e, p o o l}(t) .
$$

Value is assigned to the "energy equivalent" of all energy services every hour. The possible values and their monetary equivalents are listed in Table III. The monetary values assigned to each kWh of the "energy equivalent" are chosen arbitrarily but are loosely based on the electricity tariffs shown in Table II. That is, the value of important services is $\$ 0.60$ per $\mathrm{kWh}$ of the "energy equivalent," higher than the peak cost of electricity. For medium- and low-valued services, the monetary values are between the peak and shoulder, and shoulder and off-peak rates respectively. These assignments imply that medium-valued services may not be delivered during peak periods while low-valued services can only be delivered during the off-peak.

The hourly values of the energy services are shown in Fig. 4. The must-run service should be delivered so it has a high value at all times. From 9 AM to 5 PM, the resident does not care about the temperature inside the house because he is not present. Therefore, no value is assigned to the "energy equivalent" of space heating during this period. He also puts more value to having the room temperature near the desired value during the waking hours than during the sleeping hours.

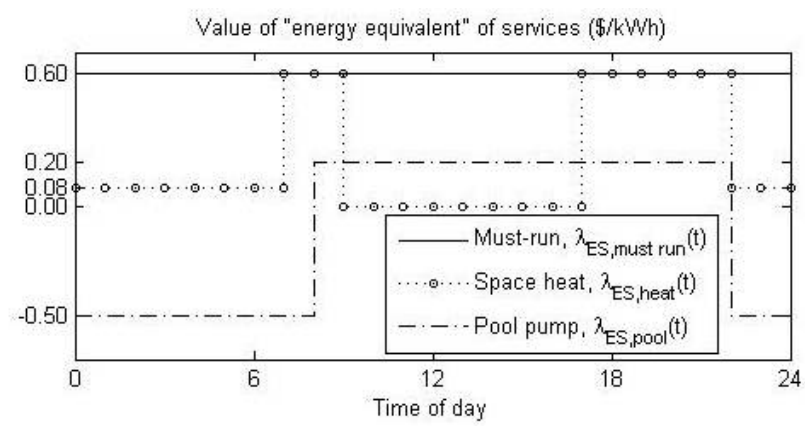

Fig. 4. Value of the "energy equivalent" of the services.

The pump could only run from 8 AM to 10 PM so medium value is assigned during this period. In Case 6 , the value of the pool pumping service during this period is reduced to low. To prevent the pump from running from $10 \mathrm{PM}$ to $8 \mathrm{AM}$, an expense value is assigned to the pumping service during that period. This implies that actually running the pump during the night is a negative benefit (or a cost).

\section{Energy Service Provision Simulation}

The objective of the simulation platform is to create a DER strategy $\boldsymbol{x}$ that would maximize the net value of the mustrun, space heating and pool pumping services. The simulation platform should find $\boldsymbol{x}$ that maximizes

$$
\sum_{t=1}^{T}\left(\begin{array}{r}
\lambda_{E S, \text { must-run }}(t) U_{E S, \text { must-run }}(t) \\
+\lambda_{E S, \text { heat }}(t) U_{E S, \text { heat }}\left(t, \boldsymbol{x}_{\text {heat }}\right) \\
+\lambda_{E S, \text { pool }}(t) U_{E S, \text { pool }}\left(t, \boldsymbol{x}_{\text {pool }}\right) \\
-\lambda_{e}(t) P_{e}(t, \boldsymbol{x})
\end{array}\right)
$$

where

$$
\boldsymbol{x}=\left[\begin{array}{lll}
\boldsymbol{x}_{\text {battery }} & \boldsymbol{x}_{\text {heat }} & \boldsymbol{x}_{\text {pool }}
\end{array}\right],
$$

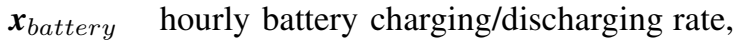

$\boldsymbol{x}_{\text {heat }}$ hourly heating power of heater,

$\boldsymbol{x}_{\text {pool }} \quad$ pool pump starting times and state $=\left[\right.$ Startime $_{1}$ Startime $_{2}$ State $_{1}$ State $\left._{2}\right]$.

The mathematical optimization problem has the following constraints:

1) The energy stored in the batteries should be within $20 \%$ to $100 \%$ of the capacity.

2) The charging and discharging rates should not exceed the maximum values.

3) The heating power should be non-negative and should not exceed the maximum value.

4) The pumping periods should not overlap.

For the space heating service, the room temperature computed using (10) should be within $1 \mathrm{C}^{\circ}$ from the desired temperature. If the room temperature is not within that range at time $t$, the value of $\lambda_{E S \text {, heat }}(t)$ in (13) is set to zero.

In the pool pumping schedule $\boldsymbol{x}_{\text {pool }}$, State St $_{i}=1$ if the $i^{\text {th }}$ 3-hour pumping period will run starting at Starttime $_{i}$, otherwise, State $_{i}=0$. The energy consumed by the pool pump $P_{e, p o o l}$ can be easily derived from $\boldsymbol{x}_{\text {pool }}$.

The total electricity imported from the grid $P_{e}(t, \boldsymbol{x})$ is computed by adding the battery charging or discharging power 
TABLE IV

SUMMARY OF RESULTS

\begin{tabular}{c|cccccc}
\hline Case number & 1 & 2 & 3 & 4 & 5 & 6 \\
\hline Battery storage & no & no & yes & no & yes & no \\
\hline Feed-in & no & no & no & yes & yes & no \\
\hline Cost, $\$$ & 4.40 & 3.60 & 3.25 & 0.84 & 0.21 & 3.30 \\
\hline Net import, $\mathrm{kWh}$ & 25.8 & 26.1 & 26.5 & 18.8 & 19.2 & 23.4 \\
\hline Total export, $\mathrm{kWh}$ & 2.0 & 0.0 & 0.0 & 7.8 & 9.5 & 0.0 \\
\hline Peak demand, $\mathrm{kW}$ & 2.6 & 2.6 & 3.0 & 2.6 & 2.7 & 2.6 \\
\hline Pump hours & 6.00 & 6.00 & 6.00 & 0.00 & 0.00 & 3.00 \\
\hline
\end{tabular}

to the energy required to deliver all services, less the output power of the PV:

$$
\begin{aligned}
P_{e}(t, \boldsymbol{x}) & =P_{e, \text { battery }}\left(t, \boldsymbol{x}_{\text {battery }}\right)+P_{e, \text { must-run }}(t) \\
& +P_{e, \text { pool }}\left(t, \boldsymbol{x}_{\text {pool }}\right)+P_{e, \text { heat }}\left(t, \boldsymbol{x}_{\text {heat }}\right) \\
& -P_{e, P V}(t) .
\end{aligned}
$$

The DER operation strategy $\boldsymbol{x}$ is solved using hybrid PSO. Real-valued PSO is used to solve for the battery charging/discharging schedule, heating power and starting times of the pool pumping periods. The start times of the pumping service are discretized by rounding them to the nearest hour. Binary PSO is used to determine the state of the pool pump (running or not) corresponding to the computed starting time. The particles are randomly initialized and the constraints are handled using a repair algorithm [14], that is, the coordinates of particles violating the constraints are corrected.

For Cases 2 to 6, 10 simulations were executed and the best solution was chosen. Each simulation used 100 particles and 200 evolutions. The parameters used are $\omega=0.7$ and $c_{1}=c_{2}=1.4$ for PSO, and $V_{\max }=5.0, \omega=1.0$, and $c_{1}=c_{2}=7.5$ for BPSO.

\section{Simulation Results and Analysis}

The simulation results are shown in Figs. 5-10 and summarized in Table IV. The average simulation time is 7.4 seconds, using Matlab R2008b, on a $2.0 \mathrm{GHz}$ Intel Pentium Dual Core CPU.

In the baseline case (Case 1), the heater and pool pump are manually controlled and it results in a total electricity cost of $\$ 4.40$ for the day under study. From 10 AM to 3 PM, the PV output exceeds the demand so the excess is exported to the grid. Since the total exported energy of $2.0 \mathrm{kWh}$ is compensated only at ToU rates, the credit is not enough to significantly reduce the total cost.

The cost of consumption is reduced by $18 \%$ when the heater and pump are scheduled (Case 2). The operation strategies are to run the pool pump an hour earlier, and preheat the house using grid and PV energy during the shoulder period and PV energy only during the peak period. The house is heated up to $24{ }^{\circ} \mathrm{C}$ and has cooled down to $22{ }^{\circ} \mathrm{C}$ by the time the resident arrives. The consequences of these strategies are the zero energy export over the entire period and the zero grid import during the first 2 hours of the peak period.

The resident is comfortable as long as the indoor temperature is at most $1 \mathrm{C}^{\circ}$ from the desired value, therefore the heater is operated such that the temperature is at the cooler level of

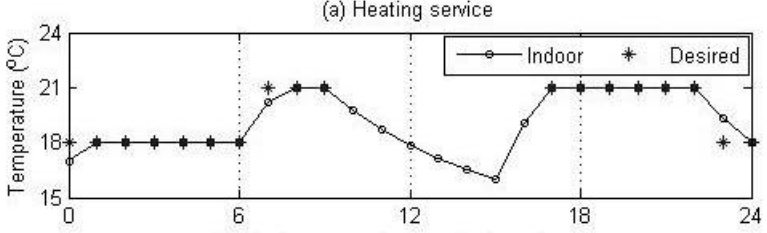

(b) Pool pump and space heater schedule

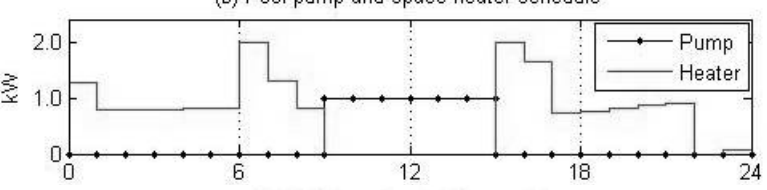

(c) Grid import and PV generation

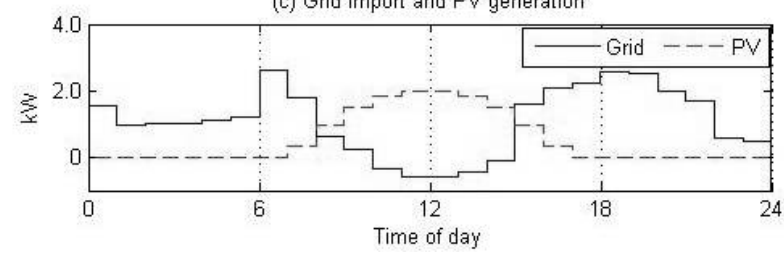

Fig. 5. Case 1: Manual control of DER, no battery storage.

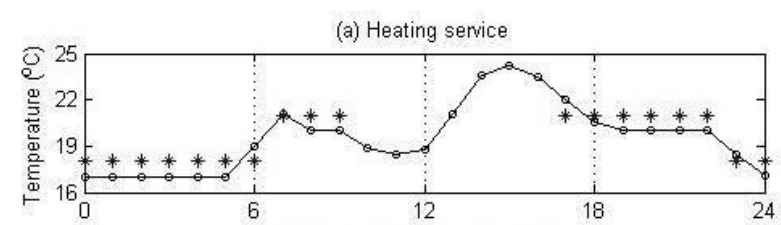

(b) Pool pump and space heater schedule

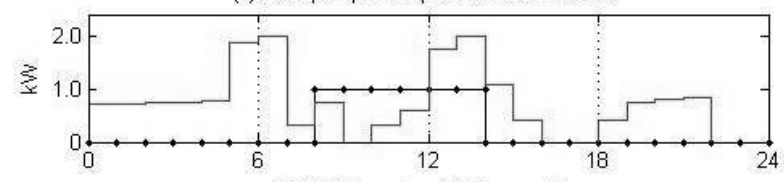

(c) Grid import and PV generation

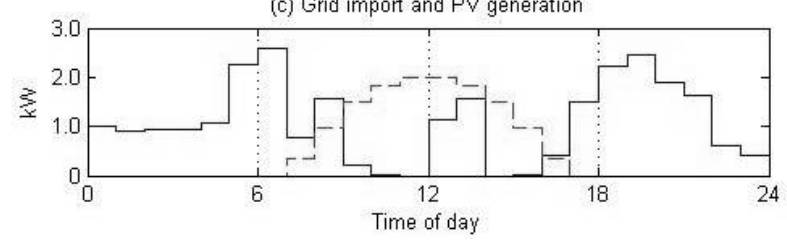

Fig. 6. Case 2: DER are scheduled, no battery storage.

the comfortable range in some hours. This resulted in a lower energy consumption of the heater. This heating strategy can also be observed in the succeeding cases.

The operation of the heater in Case 3 is almost the same as that in Case 2, causing a similar temperature profile. The pool pump is also operated from 8 AM to 2 PM. The strategy for the battery is to partially charge it during the morning off-peak, and use some of the PV output to charge it fully. The stored energy is then discharged during the peak period to displace some grid energy. The result is zero grid import during the first 3 hours of the peak period. The value added by the battery is not impressive, however, with the projected increase in retail energy prices due to the implementation of emission reduction schemes, costs associated with the achievement of renewable energy targets, and increasing network costs [15], the resident may consider installing battery storage after thorough analysis. In Australia, for example, electricity retail prices are projected to increase from $\$ 80 / \mathrm{MWh}$ in 2010 to $\$ 150 / \mathrm{MWh}$ in 2020 if 
(a) Pool pump, space heater, and battery (stored energy) schedule

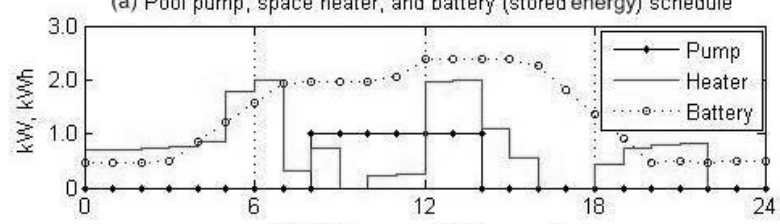

(b) Grid import and PV generation

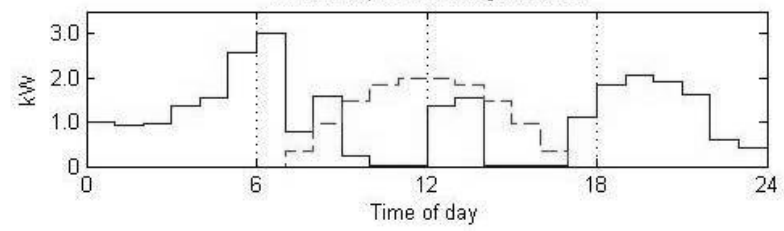

Fig. 7. Case 3: DER are scheduled, with battery storage.

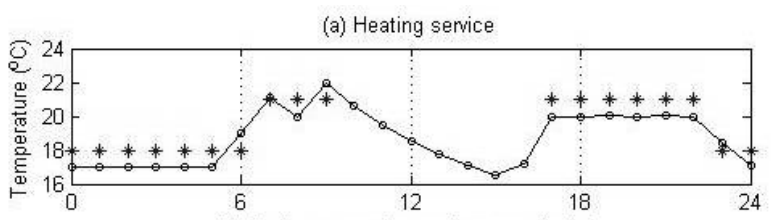

(b) Pool pump and space heater schedule

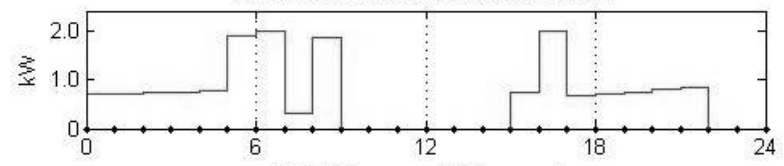

(c) Grid import and PV generation

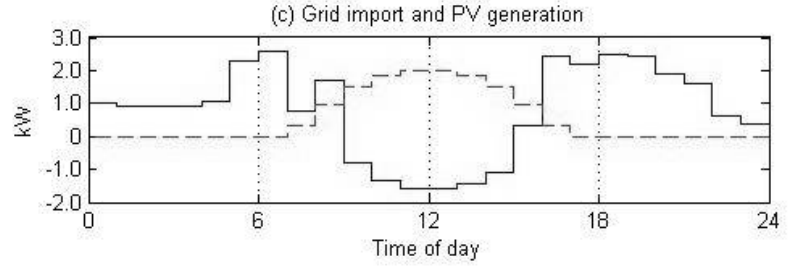

Fig. 8. Case 4: DER are scheduled, no battery storage, exported energy is remunerated at higher rates.

the Carbon Pollution Reduction Scheme will start in 2010 [16].

If net feed-in tariff is available (Case 4), the strategy is to maximize grid export while delivering only the important services. Energy is exported from 9 AM to $3 \mathrm{PM}$, and the heater is not operated during this period. The PV output in this period is also exported to the grid. For the heating service, the room is heated above the desired temperature at $9 \mathrm{AM}$ so that the energy needed to preheat the room from 3 to 5 PM is minimized. The pool pump is not operated because the benefit derived from running it is smaller compared to the compensation if the energy that would run it is exported. The $7.8 \mathrm{kWh}$ of exported energy has reduced the electricity bill to $\$ 0.84$.

If battery storage is installed (Case 5), the operation strategy is to charge it during the morning off-peak, and release the stored energy during the energy export period. The energy export increased to $9.5 \mathrm{kWh}$ and the electricity bill is reduced to $\$ 0.21$. In this case, it is potentially more plausible to install battery storage because the value it brings to the resident is higher. The heating power is identical to that in Case 4, and the pool pump is also not operated.

In Case 6, the reduced value of the pool pumping service has resulted in the reduction of the number of hours of which (a) Pool pump, space heater and battery (stored energy) schedule

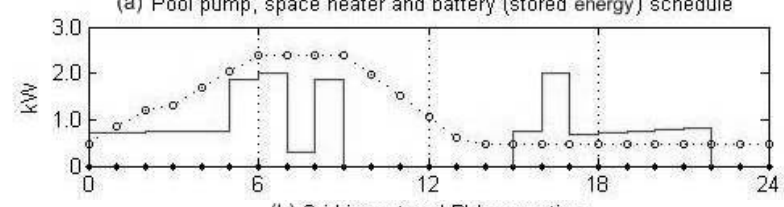

(b) Grid import and PV generation

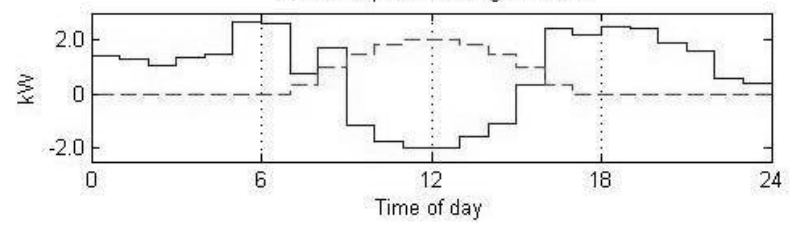

Fig. 9. Case 5: DER are scheduled, with battery storage, exported energy is remunerated at higher rates.

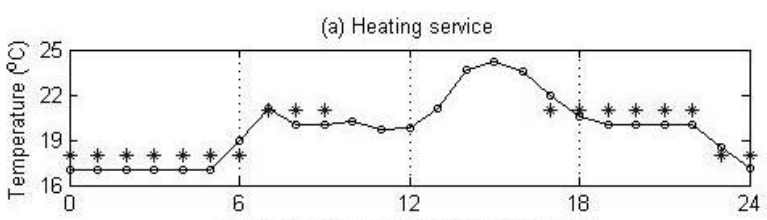

(b) Pool pump and space heater schedule

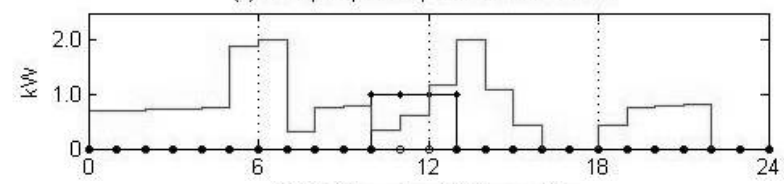

(c) Grid import and PV generation

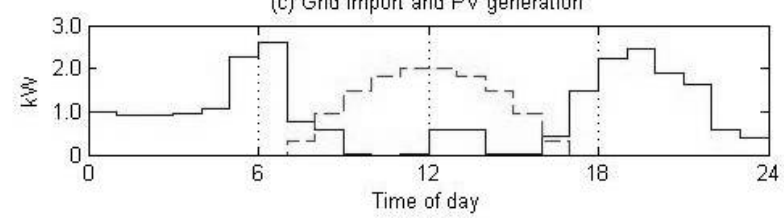

Fig. 10. Case 6: DER are scheduled, no battery storage, value of pool pumping service is reduced from medium to low.

it is run. Only 3 hours of the desired 6 hours of pool pumping is scheduled. Because of the PV output, the resident was able to benefit from the pumping service although its value is lower than the prevailing electricity rate.

The case study was able to demonstrate that the net benefit derived from energy services may be improved by scheduling the operation of available DER. The simulation platform was able to reduce the cost of consumption by taking advantage of the heat storage capability of air: the house could be preheated when energy rates are low so the space heating service required at a later time could be provided. It was able to take advantage of the net feed-in rates by maximizing the amount of energy export. It was also able to determine if the pump should operate and at what times based on the value assigned to the pool pumping service and the potential cost of its provision. The pumping service is entirely postponed when feed-in tariff is available, and only part of it is delivered when its value is reduced. In two cases, it was able to quantify the benefit if battery storage is installed.

\section{CONCLUSION}

The traditional method of delivering energy services may be improved by recognizing that different energy services have 
different values to the user. The assignment of different levels of benefit to different energy services has transformed energy service provision from cost minimization to net benefit maximization. This approach tends to prioritize the provision of important or high-valued services and opens up the possibility of postponing or cancellation of low-valued services. We have demonstrated that by using this approach, the provision of services may be economically improved. This approach may be adopted when implementing direct load control demandside management programs.

In this paper, we demonstrated that the proposed energy service modeling technique can capture and represent temporal variations of its demand and value. By putting value to the energy that realizes an energy service, we were able to differentiate it from the actual energy consumption of the end-use equipment that delivers the service. The distinction between the energy that realizes the service and the actual energy consumption is important because the presence of passive storage implies that there could be temporal mismatch between them. That is, energy consumption could occur ahead of the actual service utilization.

We used the modeling technique with a novel energy service simulation platform to improve services delivery by maximizing the net benefit due to their provision. The simulation platform was able to maximize the value of the required services while minimizing the cost of energy consumption by proposing a strategy, or a schedule, for how available DER should be operated. The simulation platform takes advantage of the temporal mismatch between energy consumption and the energy that realizes a service, the availability of flexible services and active storage options, and the temporal variation of the cost of electricity. In the presented smart home case study, it was able to suggest effective strategies under different tariff schemes and different values assigned to services. It was able to schedule the DER to reduce the cost of consumption, and to maximize energy export when net feed-in tariff is available. It was able to postpone low-valued services when the cost of provision exceeds the benefit from having the service.

The creation of the strategy is a non-linear, non-convex and non-continuous mathematical optimization problem. The chosen optimization tool, particle swarm optimization, was able to generate effective strategies within short computation times.

\section{REFERENCES}

[1] R. Haas, N. Nakicenovic, A. Ajanovic, T. Faber, L. Kranzi, A. Muller and G. Resch, "Towards sustainability of energy systems: A primer on how to apply the concept of energy services to identify necessary trends and policies," Energy Policy, vol. 36, no. 11, pp. 4012-4021, Nov. 2008.

[2] H. Outhred, "Electricity industry restructuring in Australia: underlying principles and experience to date," in Proc. 40th Annual Hawaii International Conference on System Sciences, 2007, p. 125.

[3] A. Lovins, E. Datta, T. Feiler, K. Rabago, J. Swisher, A. Lehmann and K. Wicker, Small is Profitable: The Hidden Economic Benefits of Making Electrical Resources the Right Size. Snowmass, CO: Rocky Mountain Institute, 2002.

[4] S. Abras, S. Pesty, S. Ploix and M. Jacomino, "An anticipation mechanism for power management in a smart home using multi-agent systems," presented at the 3rd International Conference on Information and Communication Technologies: From Theory to Applications, April 2008.
[5] C. Marnay, G. Venkataramanan, M. Stadler, A. Siddiqui, R. Firestone and B. Chandran, "Optimal technology selection and operation of commercial-building microgrids," IEEE Trans. Power Systems, vol. 23, no. 3, pp. 975 - 982, Aug. 2008

[6] J. Kennedy and R. Eberhart, "Particle swarm optimization," in Proc. 1995 IEEE International Conference on Neural Networks, vol. 4, pp. 1942 - 1948.

[7] J. Kennedy and R. Eberhart, "A discrete binary version of the particle swarm algorithm," in Proc. 1997 IEEE International Conference on Systems, Man and Cybernetics, vol. 5, pp. 4104 - 4108.

[8] K. T. Chaturvedi, M. Pandit, and L. Srivastava, "Self-organizing hierarchical particle swarm optimization for non-convex economic dispatch," IEEE Trans. Power Systems, vol. 23, no. 3, pp. 1079 - 1087, Aug. 2008.

[9] T. O. Ting, M. V. C. Rao, and C. K. Loo, "A novel approach for unit commitment via an effective hybrid particle swarm optimization," IEEE Trans. Power Systems, vol. 21, no. 1, pp. 411 - 418, Feb. 2006.

[10] P.-H. Chen, "Pumped-storage scheduling using evolutionary particle swarm optimization," IEEE Trans. Energy Conversion, vol. 23, no. 1, pp. 294 - 301, Mar. 2008.

[11] Energy Australia NSW [Online]. Available: http://www.energy.com.au/ energy/ea.nsf/ Content/NSW+home.

[12] Tackling Climate Change in South Australia, South Australia Solar Feedin Scheme [Online]. Available: http://www.climatechange.sa.gov.au/ index.php?page $=$ feed-in-scheme

[13] H. Sauer, R. Howell, and W. Coad, Principles of Heating, Ventilating and Air Conditioning. Atlanta, GA: ASHRAE, 2001.

[14] C. A. Coello, "Theoretical and numerical constraint-handling techniques used with evolutionary algorithms: a survey of the state of the art," in Computer Methods in Applied Mechanics and Engineering, vol. 191, no. 11-12, 4 January 2002, pp. 1245 - 1287.

[15] The Australian Energy Market Commission, "Survey of evidence on the implications of climate change policies for energy markets." Sydney, Australia, December 2008.

[16] McLennan Magasanik Associates, "Impacts of the carbon pollution reduction scheme on Australia's electricity markets," Australia Federal Treasury, Canberra, Australia, December 2008.

Michael Angelo Pedrasa received his B.S. and M.S. Electrical Engineering degrees at the University of the Philippines and is currently sitting his Ph.D. at the University of New South Wales in Sydney, Australia. His studies at the UNSW are supported by the University of the Philippines (UP-DSF) and the Philippines Department of Science and Technology (DOST-ERDT). His research interest are power system optimization and integration of distributed energy resources to electric power systems.

Ted Spooner received his BE and ME degrees from the University of New South Wales in 1970 and 1973 and has been a senior lecturer at The University of New South Wales in the School of Electrical Engineering and Telecommunications since 2002. His research interests are in renewable energy applications and power electronics. He was project leader for Australia's renewable energy systems testing laboratory now known as RESLab. $\mathrm{He}$ is currently a chair of Australian Standards Committee responsible for renewable energy systems. He is also the Australian representative on the International Electrotechnical Commission's (IEC) technical committee TC82 for Photovoltaics working in the area of systems.

Dr. Iain MacGill is a Senior Lecturer in the School of Electrical Engineering and Telecommunications at the University of New South Wales, and Joint Director for the University's interdisciplinary Centre for Energy and Environmental Markets. Iain's teaching and research interests include electricity industry restructuring, sustainable energy technologies with a particular focus on distributed resources and energy policy. 